\title{
The effect of interspecific competition on the temporal dynamics of Aedes albopictus and Culex pipiens
}

Giovanni Marini ${ }^{1,2^{*}+}$ (D), Giorgio Guzzetta ${ }^{3+}$, Frederic Baldacchino ${ }^{2}$, Daniele Arnoldi ${ }^{2}$, Fabrizio Montarsi ${ }^{4}$, Gioia Capelli', Annapaola Rizzoli², Stefano Merler ${ }^{3}$ and Roberto Rosà ${ }^{2}$

\begin{abstract}
Background: Aedes albopictus and Culex pipiens larvae reared in the same breeding site compete for resources, with an asymmetrical outcome that disadvantages only the latter species. The impact of these interactions on the overall ecology of these two species has not yet been assessed in the natural environment. In the present study, the temporal patterns of adult female mosquitoes from both species were analysed in north-eastern Italy, and substantial temporal shifts between abundance curves of Cx. pipiens and Ae. albopictus were observed in several sites. To understand which factors can drive the observed temporal shifts, we developed a mechanistic model that takes explicitly into account the effect of temperature on the development and survival of all mosquito stages. We also included into the model the effect of asymmetric interspecific competition, by adding a mortality term for $C x$. pipiens larvae proportional to the larval abundance of Ae. albopictus within the same breeding site. Model calibration was performed through a Markov Chain Monte Carlo approach using weekly capture data collected in our study sites during 2014 and 2015.
\end{abstract}

Results: In almost half of observation sites, temporal shifts were due to competition, with an early decline of Cx. pipiens caused by the concurrent rise in abundance of its competitor, and this effect was enhanced by higher abundance of both species. We estimate that competition may reduce $C x$. pipiens abundance in some sites by up to about $70 \%$. However, in some cases temporal shifts can also be explained in the absence of competition between species resulting from a "temporal niche" effect, when the optimal fitness to environmental conditions for the two species are reached at different times of the year.

Conclusions: Our findings demonstrate the importance of considering ecological interactions and, in particular, competition between mosquito species in temperate climates, with important implications for risk assessment of mosquito transmitted pathogens, as well as the implementation of effective control measures.

Keywords: Computational model, Mosquito population dynamics, Species interaction, Temporal shift, Vector abundance

\section{Background}

A fundamental concept in ecology is that competition for limited resources can take place between individuals of the same species, i.e. intraspecific competition, or between individuals of different species, i.e. interspecific competition. In interspecific competition, individuals of

\footnotetext{
* Correspondence: giovanni.marini@fmach.it

${ }^{\dagger}$ Equal contributors

'Department of Mathematics, University of Trento, Trento, Italy

${ }^{2}$ Department of Biodiversity and Molecular Ecology, Research and Innovation

Centre, Fondazione Edmund Mach, San Michele all'Adige, Trento, Italy

Full list of author information is available at the end of the article
}

different species compete for the same resource (e.g. food or living space) therefore limiting resource availability for the other species. Such competition could lead to the exclusion of the weaker species but also to coexistence equilibrium via different mechanisms, such as differential resource use and spatial or temporal variations in habitat conditions [1, 2].

Among mosquito species, interspecific competition plays a key role in structuring the community at the larval stage in water-filled containers [3]. In Europe, Aedes albopictus and Culex pipiens are two of the most widely 
spread mosquito species [4-6]. While $C x$. pipiens is indigenous, Ae. albopictus is native to Asia and was introduced in several European countries at the end of the last century [5]; since then, Ae. albopictus rapidly spread in urban and suburban environments, occupying a habitat already exploited by $C x$. pipiens. These two species are vectors of many arboviruses. Ae. albopictus can transmit dengue, chikungunya, Zika and West Nile viruses [7-12], while $C x$. pipiens is the most important vector of West Nile virus in Europe [13]. Local vector abundance, which drives the pathogen-transmission dynamics $[14,15]$, might be influenced by interspecific competition. Furthermore, larval competition might have indirect effects on epidemiological risks by altering mosquito-virus interactions in adult females [16]; different Aedes mosquitoes (including Ae. albopictus, Ae. aegypti and Ae. triseriatus) bred in conditions of nutritional stress imposed by the interspecific competition were more susceptible to infection and more able to transmit various pathogens such as dengue, Sindbis and LaCrosse viruses [17-19]. In addition, adult survival, and consequently the length of infectious period for infected females (i.e. their vectorial capacity), might be influenced by interspecific interactions occurred at the larval stage $[20,21]$. Therefore, competition may have important consequences on the epidemiology of mosquito-borne infections and their potential control strategies [3].

Previous laboratory studies show that Ae. albopictus is a strong competitor against other species; Ae. albopictus larvae have been shown to negatively affect the growth and survival of larvae from other mosquito species bred in the same site, including Ae. aegypti [22, 23], Ae. japonicus [24], Ae. triseriatus [25, 26], Ae. koreicus (F. Baldacchino, unpublished observations) and Culex pipiens $[27,28]$. Aedes albopictus larvae, on the other hand, were substantially unaffected by the presence of $C x$. pipiens larvae. This asymmetric interspecific competition has been attributed to a higher efficiency of Ae. albopictus in converting food to biomass [27]. The strength of competition effects has been shown to depend on food resource types [27] and temperature, with a maximal effect on $C x$. pipiens larval mortality observed at temperatures above $25^{\circ} \mathrm{C}$ [27].

In nature, Ae. albopictus and Cx. pipiens can exploit common water-filled containers as larval habitats. Generally, Ae. albopictus prefers ovipositing in small natural and artificial containers, while $C x$. pipiens prefers larger water bodies [27, 29]. However, these two species can share medium size containers. In northern Italy, during entomological surveys in the summer of 1996 and 1997, Ae. albopictus and Cx. pipiens were detected together in $67 \%$ of larval habitats, especially drums, buckets, catch basins and tires [27]. Thus, coexistence between these two species could be shaped by both interspecific competition and niche differentiation involving temporal and spatial factors $[2,3]$.

The temporal patterns of local populations of Ae. albopictus and $C x$. pipiens in northern Italy can be highly variable depending on climate and landscape [30,31], but generally $C x$. pipiens is active earlier than Ae. albopictus $[27,32]$. Culex pipiens larvae appear in springtime and peak in July, while Ae. albopictus larvae appear several weeks after $C x$. pipiens and peak in September [27]. Different temporal profiles may be driven by different life history strategies and patterns of survival, oviposition and egg hatching under variable environments [27, 28]. In the case of $C x$. pipiens, only adult females undergo diapause, and shortening photoperiods induce diapause in a growing number of newly emerged adult females [33, 34]. Early in the mosquito breeding season, overwintering females of $C x$. pipiens begin to lay eggs on water surface. In contrast, Ae. albopictus overwinters as diapausing eggs [29], which hatch several weeks after $C x$. pipiens eggs; then newly emerged Ae. albopictus females lay eggs above the water line, and hatching is induced by submergence after precipitations. This asynchrony in hatching between the two species allows $C x$. pipiens larvae to develop in the absence of Ae. albopictus and provides to $C x$. pipiens a refuge from competition early in the season [28]. Furthermore, high temperatures observed in summertime decrease $C x$. pipiens adult survival $[35,36]$, while Ae. albopictus is better adapted to warmer conditions [37]. Therefore, environmental conditions can create a "temporal niche" effect, allowing a shift in optimal breeding seasons of the two species. Nonetheless, the "temporal niche" effect is not always sufficient to explain the observed temporal profiles of competing mosquito species [2].

Disentangling the ecological mechanisms that drive mosquito population dynamics might be difficult with a simple statistical analysis of the observed capture pattern. However, mechanistic models incorporate a range of biological processes that drive mosquito population dynamics. Therefore, they are more suitable to investigate the main determinants of dynamical patterns, such as the effect of "temporal niches" and interspecific competition. Several mechanistic models have been used to describe the population dynamics of single mosquito species, including Ae. albopictus [38-40] and Cx. pipiens [41-43]. In this study, we describe and interpret, in a robust mathematical framework, observed differences in temporal patterns of Ae. albopictus and Cx. pipiens, aiming to disentangle the contribution of the "temporal niche" effect and interspecific competition on their population dynamics.

To this aim, we develop a mathematical model that describes temporal variations of population dynamics of both species and allows for interspecific interactions at 
the larval stages, as previously evaluated in laboratory conditions. To the best of our knowledge, this is one of the first efforts to assess the impact of Ae. albopictus and $C x$. pipiens ecological interactions in natural conditions.

\section{Methods}

\section{Study area and mosquito data}

The study was carried out in the provinces of Belluno $\left(46^{\circ} 08^{\prime} 27^{\prime \prime} \mathrm{N}, 12^{\circ} 12^{\prime} 56^{\prime \prime} \mathrm{E}\right)$ and Trento $\left(46^{\circ} 04^{\prime} 00^{\prime \prime} \mathrm{N}, 11^{\circ}\right.$ $\left.07^{\prime} 00^{\prime \prime} \mathrm{E}\right)$, Northern Italy. This mountainous area covers a large part of the Dolomites and the Southern Alps. The climate is temperate-oceanic with four main areas: sub-Mediterranean (close to Lake Garda with mild winters), subcontinental (the main valleys with more severe winters), continental (the alpine valleys) and alpine (the areas above the tree line) [44].

We performed entomological surveillance of several mosquito species in the provinces of Trento and Belluno during 2014 and 2015. Mosquitoes were collected using Biogents Sentinel traps (BG trap; Biogents AG, Regensburg, Germany) baited with commercial lures from the same producer and $\mathrm{CO}_{2}$ from dry ice. The $\mathrm{BG}$ trap has been demonstrated to collect a great variety of species and is efficient for both Ae. albopictus and Cx. pipiens when baited with $\mathrm{CO}_{2}$, especially in an urban environment [45]. Trapping locations were chosen within three municipalities of Belluno province (Belluno, Feltre, Santa Giustina) and three of Trento province (Borgo Valsugana, Riva del Garda, Trento) (see Fig. 1).

A total of 73 BG traps were located within urban and peri-urban areas at altitudes ranging from $75 \mathrm{~m}$ to about $640 \mathrm{~m}$ above sea level. As recommended by the manufacturer, traps were placed in shaded positions sheltered from wind and rainfall. In 2014, 39 traps were positioned while in 2015 the number of traps was reduced to $34 ; 28$ trapping sites where shared among the two years. BG traps ran for $24 \mathrm{~h}$, approximately every two weeks, from the end of April to the beginning of November.
Mosquito sex and species were identified using taxonomic keys [46]. The number of capture sessions differed from trap to trap because of logistic reasons, ranging from a minimum of 8 to a maximum of 15 .

\section{Delay analysis}

We analysed temporal shifts between pairs of time series of captured female adults of $C x$. pipiens and Ae. albopictus, observed at the same site and year. To this aim, we estimate the time $\operatorname{lag} T$ between the two time series at which the cross-correlation function $\rho$ reaches its maximum (see [47] for details):

$$
T=\operatorname{argmax}_{\tau} \rho_{X Y}(\tau)=\underset{\tau}{\operatorname{argmax}} \sum_{t=1}^{m} X[t] Y[t+\tau]
$$

More specifically, $\rho_{X Y}[\tau]$ is the cross-correlation function, $X$ and $Y$ are the time series for $C x$. pipiens and Ae. albopictus respectively, $m$ is the length of the time series expressed in weeks. $T$ measures the time lag (in weeks) between the two time series and it is labelled throughout the manuscript as the "interspecific delay" between Ae. albopictus and $C x$. pipiens temporal dynamics.

\section{Environmental data}

Original land surface temperature (LST) data were obtained from the MODIS version 5 LST products MOD11A1 and MYD11A1 [48]. We used the average daily temperature and a spatial resolution of $250 \mathrm{~m}$ [49].

There was a striking difference in recorded temperatures between the two considered years (Fig. 2a, b); specifically, for the 28 sites sampled in both years, the difference in the average daily temperature during summer months (July to September) between 2014 and 2015 (Fig. 2c) is close to $5{ }^{\circ} \mathrm{C}$, with average observed temperatures of $20.6{ }^{\circ} \mathrm{C}$ and $25.5{ }^{\circ} \mathrm{C}$ for the two years, respectively. For comparison, the average temperature for this period computed over years $2004-2013$ is about $21.9^{\circ} \mathrm{C}$ [50], so that the two years of study represent the

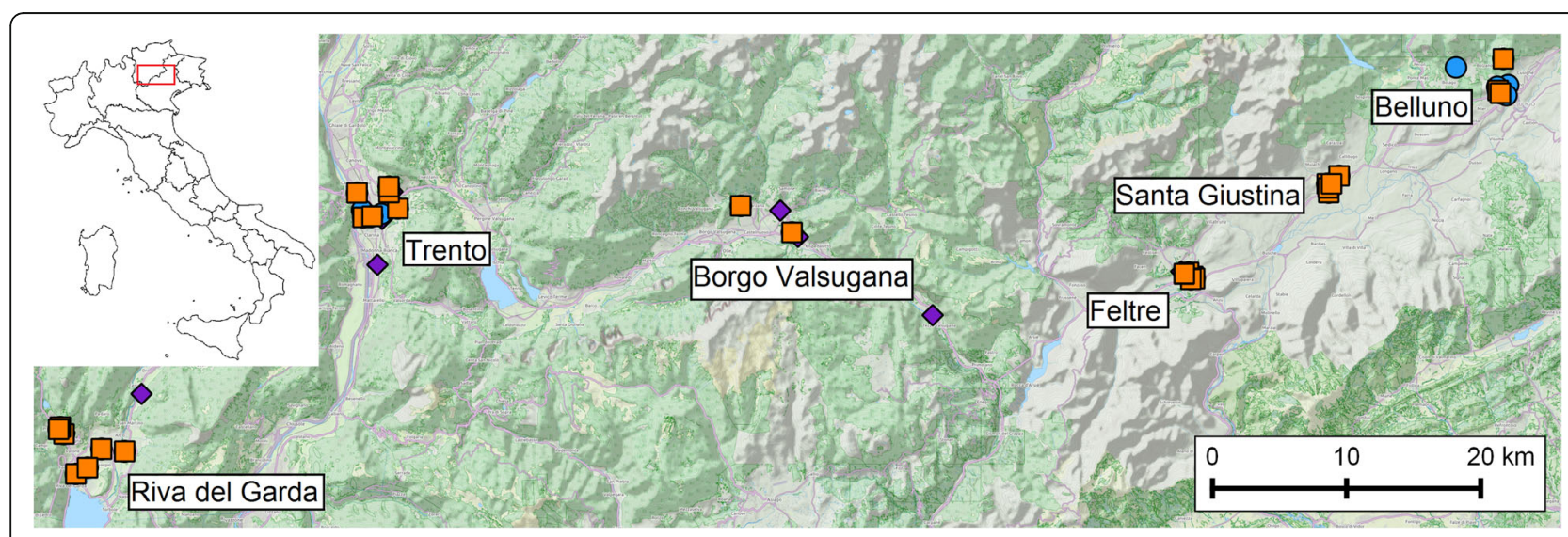

Fig. 1 Map of the study area. Selected trap locations (purple diamonds: 2014; blue circles: 2015; orange squares: both years) 

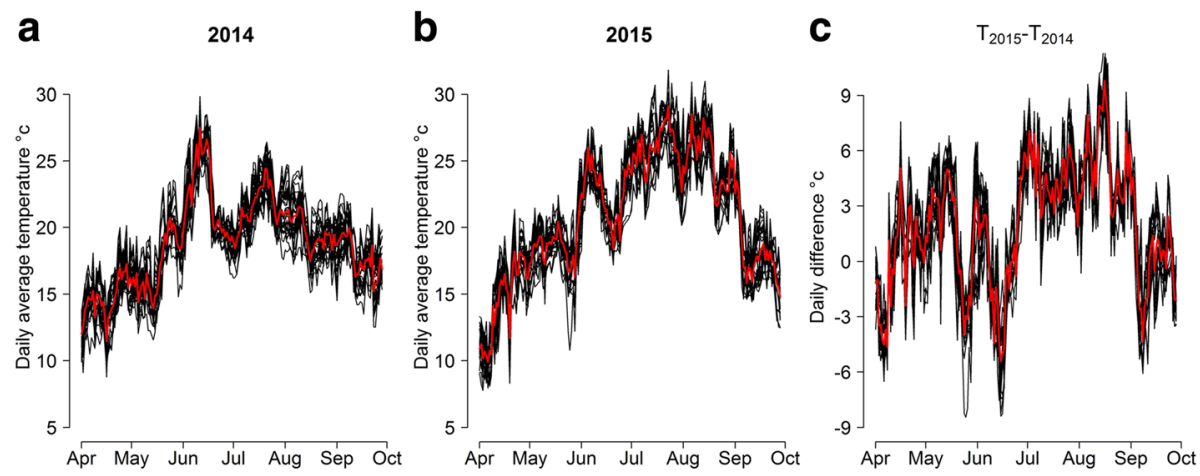

Fig. 2 Daily temperature at study sites. a Daily average temperatures from the 39 trap locations in 2014 (black lines) and average across all sites (red line); b daily average temperatures from the 34 trap locations in 2015 (black lines) and average across all sites (red line); c daily average temperature difference between 2015 and 2014 from the 28 sites represented in both years

extremes of a wide range of possible temperature scenarios. Daylight lengths for the centroid of the study region during the considered period were obtained from the US Naval Observatory [51].

\section{Population model}

We developed a mathematical model of the abundance of the two vector species based on the Ae. albopictus model proposed in $[39,52,53]$ and on the $C x$. pipiens model proposed in [43]. The original models account for the population dynamics of each species by considering temperature-dependent development and mortality rates and intraspecific larval density dependent factors; the model for $C x$. pipiens also includes a photoperioddependent diapause rate for pupae. Here, we include the effect of asymmetric interspecific competition, by adding a mortality term for $C x$. pipiens larvae proportional to the larval abundance of Ae. albopictus in the same site. Given that the effect of interspecific competition weakens for temperatures below $20^{\circ} \mathrm{C}$ [27], we set the competition coefficient to zero for average daily temperatures less than $15{ }^{\circ} \mathrm{C}$, and to a constant value, estimated via model calibration, otherwise.

The model was calibrated using a Markov Chain Monte Carlo (MCMC) approach applied to the Poisson likelihood of observing the empirical capture data, given the model-predicted abundance. The model has five free model parameters: two daily capture rates of adult mosquitoes (one for Ae. albopictus and one for Cx. pipiens); an intraspecific larval density dependent factor for each species, representing the availability of suitable breeding sites and food resources at a given site; and the interspecific larval competition coefficient, which increases the mortality of $C x$. pipiens larvae proportionally to the abundance of Ae. albopictus larvae. The posterior distribution of parameters was obtained by a random-walk Metropolis-Hastings sampling [54], using uniform prior distributions and normal jumps.
To evaluate the hypothesis of interspecific competition, we calibrated a simplified model representing the assumption of independent populations, where the larval competition coefficient was fixed to zero. We then compared the goodness of fit of the two models (with and without competition) using the Deviance Information Criterion (DIC) $[55,56]$. The model including competition was preferred to the model with independent populations when its DIC value was lower by a minimum threshold, which was conservatively set to four (compared to a minimum recommended threshold of 2 [55]).

All relevant details on model equations, calibration procedure, model selection and sensitivity analysis of results with respect to model selection criteria are reported in the Additional file 1: Database 1 (see sections "Model calibration" and "DIC and AIC analysis").

\section{Results}

The total number of trapped female Ae. albopictus and $C x$. pipiens in all sites and years was 4,566 and 8,362, respectively. Capture data are presented in the Additional file 2: Database S2. As shown in Fig. 3, Cx. pipiens abundance was similar between the two years (54\% of total captured in 2014) while the majority of Ae. albopictus were captured in 2015 (69\% of total). This is likely because 2015 was warmer and therefore more suitable for Ae. albopictus.

Culex pipiens shows different patterns between the two considered years. In fact, in 2015 Cx. pipiens abundance starts declining earlier in the season, in conjunction with the increase of Ae. albopictus abundance, while in 2014 the two species show a more synchronous pattern (Fig. 3). On the other hand, temporal dynamics of Ae. albopictus do not show substantial inter-annual differences (Fig. 3).

Figure 4 presents the distribution of interspecific delays computed over all available time series. We can note that temporal profiles of Ae. albopictus are delayed, with respect to $C x$. pipiens from the same site and year, by more than 4 weeks (29 days) on average; a higher delay 


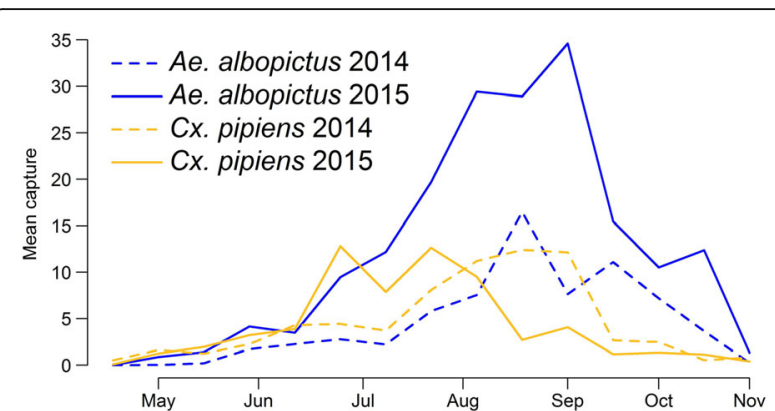

Fig. 3 Mosquito temporal dynamics. Number of adult female mosquito captures for the two species (Ae. albopictus in blue and Cx. pipiens in yellow), averaged over all sites, for 2014 and 2015 (dashed and continuous lines respectively)

was recorded in 2015 (37 days) with respect to 2014 (22 days) ( $t$-test $t=-1.9912, d f=69.597, \quad P=0.050)$ (Fig. 4). Figure 5 illustrates two examples of observed capture patterns associated with an average delay of four weeks (Fig. 5a) and no delay (Fig. 5b).

For 29 time series (around 40\%) the model with competition assumption was better $(\triangle \mathrm{DIC}>4)$ at explaining the observed capture dynamics; of these, the large majority (22) were time series from 2015 (see Table 1). On the other hand, 44 time series (around 60\%) were better described $(\triangle \mathrm{DIC}<4)$ by the model without the competition assumption; of these, 32 were time series observed in 2014 (Table 1). An overall comparison of selected model fits for all time series can be seen in the Additional file 3: Figure S1.

In Fig. 6, we show the distribution of interspecific delays aggregated by the selected model (competition $v s$ independent populations) and by year. In sites with competition, Ae. albopictus capture patterns had a systematic and large positive delay with respect to $C x$. pipiens (average 51 days considering the two years combined). Conversely, in sites where the independent population model prevailed, the average interspecific delay

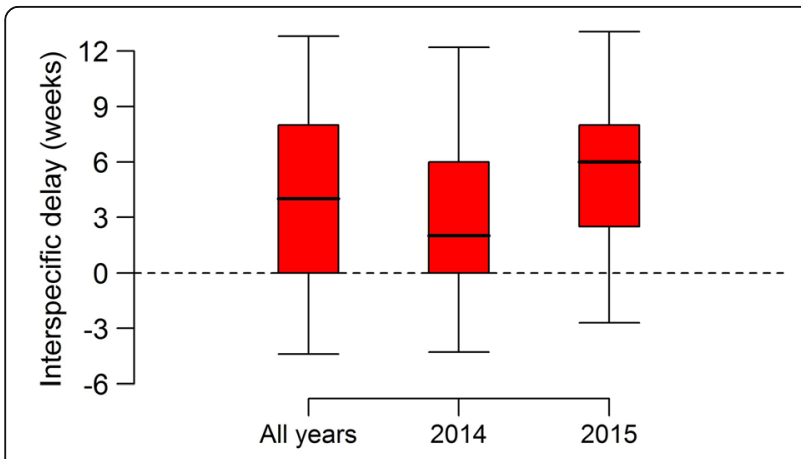

Fig. 4 Interspecific delay. The interspecific delay (in weeks, median, quartiles and 95\% quantiles) computed for Ae. albopictus and Cx. pipiens capture patterns. Distributions are shown for all time series combined and aggregated by year

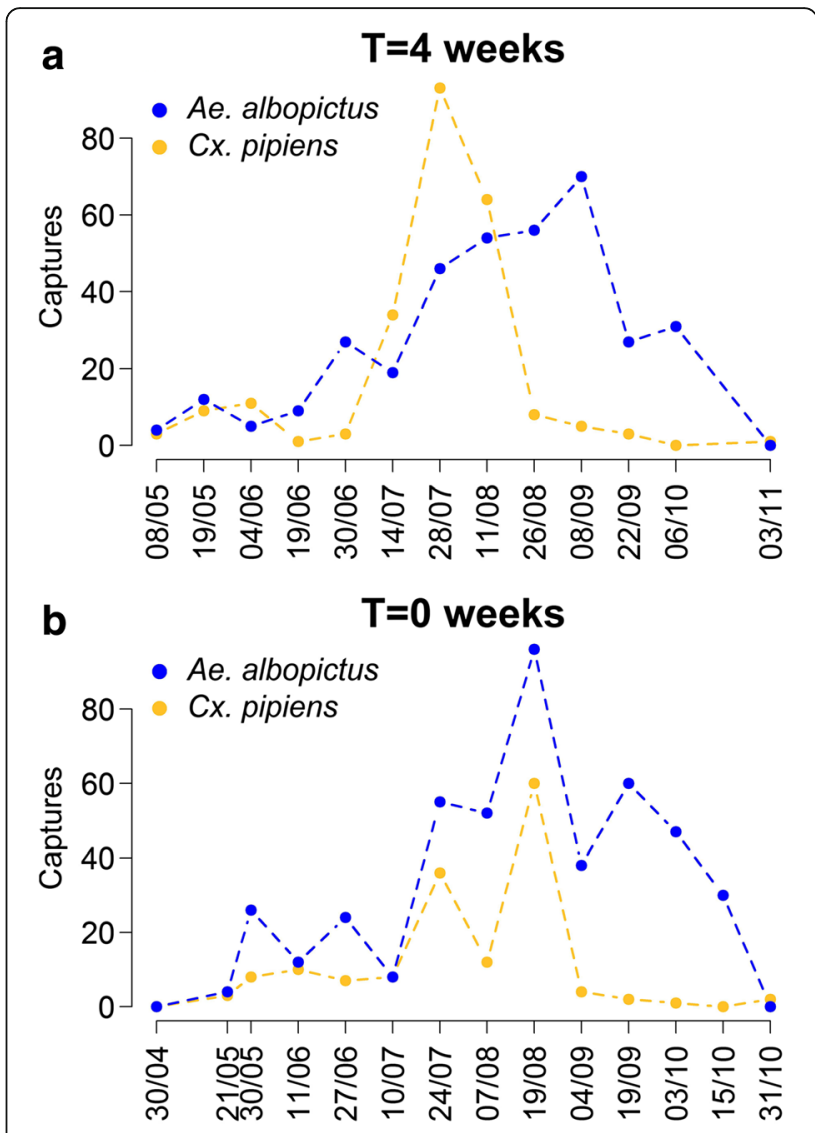

Fig. 5 Two examples of recorded temporal patterns with different interspecific delay. $T=4$ weeks (a), no delay, i.e. $T=0$ (b). Aedes albopictus (blue) and $\mathrm{Cx}$. pipiens (yellow) recorded captures from two datasets

was lower (14 days considering the two years combined). There were no significant differences between average delays from the same group and different years.

We analysed the average numbers of mosquitoes captured per session and site, aggregated by selected model and year (Fig. 7). In 2014, when competition was rare, abundances of $C x$. pipiens and Ae. albopictus were not significantly different within competition time series compared to those without; however, in 2015 competition was much more common, and both Ae. albopictus and $C x$. pipiens were significantly more abundant within competition sites $(t$-test $t=-3.2873, d f=23.758, P=0.003)$. This result suggests that high mosquito densities might have increased the chance of competition in 2015, possibly because of the increased likelihood of shared oviposition sites. Interestingly, despite the higher mortality of $C x$. pipiens larvae in competition sites, we did not find an overall reduction in Cx. pipiens captures in 2015 respect to 2014 within competition sites. This seemingly counterintuitive result can be better interpreted by considering temporal dynamics (Fig. 3): in the early part of the 2015 season, $C x$. pipiens were much more abundant than in the same period of 2014, because of improved environmental 
Table 1 Number of time series by year and selected model. Time series, classified according to model selection based on the Deviance Information Criterion, are shown for grouped and separated years. Percentages are computed by row

\begin{tabular}{llll}
\hline & All time series & Competition (\%) & $\begin{array}{l}\text { Independent } \\
\text { populations (\%) }\end{array}$ \\
\hline All years & 73 & $29(40)$ & $44(60)$ \\
2014 & 39 & $7(18)$ & $32(82)$ \\
2015 & 34 & $22(65)$ & $12(35)$ \\
\hline
\end{tabular}

conditions; however, with the rapid expansion of Ae. albopictus, the 2015 abundance of Cx. pipiens fell briskly, offsetting the advantage of the first part of the season. Considering only sites with competition, the model predicted a reduction for the average $C x$. pipiens abundance by $49.2 \%$ (95\% quantiles: $14-74 \%$ ), compared to corresponding simulations obtained with independent populations. The onset of competition effects (defined as the first date at which relative differences between the $C x$. pipiens populations predicted by models with and without competition exceed 10\%) ranged from the end of April to the middle of July, with median centred on the first ten days of June.

\section{Discussion}

In this study, we analysed time series of Ae. albopictus and $C x$. pipiens captures in northern Italy. The observed dynamics of the two species show, in several cases, a marked misalignment in temporal patterns, with a delay of Ae. albopictus abundance patterns with respect to $C x$. pipiens. We showed that these temporal shifts could be explained by two alternative mechanisms: temporal niche effects and asymmetric interspecific larval competition. Under the assumption that the two populations do not interfere with each other in a given area, a temporal niche effect may occur, depending on environmental variables (e.g. temperature, photoperiod), when the two species reach their peak of abundance at different times of the year. On the other hand, in general, competition causes more pronounced delays between the dynamics of the two species. That is due to an increase of Cx. pipiens larval mortality in the presence of Ae. albopictus within the same breeding site, inducing an anticipated decline of $C x$. pipiens adult population.

An asymmetric competition between Ae. albopictus and $C x$. pipiens has been observed and replicated in several laboratory experiments $[27,28]$, but its importance in the natural environment has not been assessed before. According to our modelling results, asymmetric interspecific competition explained the seasonal patterns of $C x$. pipiens well in many sampling sites. Specifically, in 2015 the model with competition assumption better described empirical observations in $65 \%$ of the considered sites, compared to only $18 \%$ in 2014 . Our results suggest that this difference was associated with higher temperatures in 2015 than in 2014, which caused both a direct increase in the competition effect [27] and an increase of Ae. albopictus densities due to a considerable reduction in the development time of immature stages [37]. High Ae. albopictus densities may increase the chance of oviposition in containers shared with $C x$. pipiens [21]. In addition, drier conditions observed in summer 2015 (about $122 \mathrm{~mm}$ total precipitations on average, compared to $355 \mathrm{~mm}$ in 2014 [50]) likely reduced the number of small rain-filled containers available for Ae. albopictus, pushing females to oviposit in medium containers with the presence of $C x$. pipiens larvae. Thus, the larval habitats of $A e$. albopictus and $C x$. pipiens might have overlapped more under such conditions, leading to a greater frequency of interspecific competition [27]. Abiotic factors in 2015 were also more favourable for Cx. pipiens, but the adverse effects of competition strongly limited their increase with respect to 2014. We estimated a relative reduction of $C x$. pipiens abundance

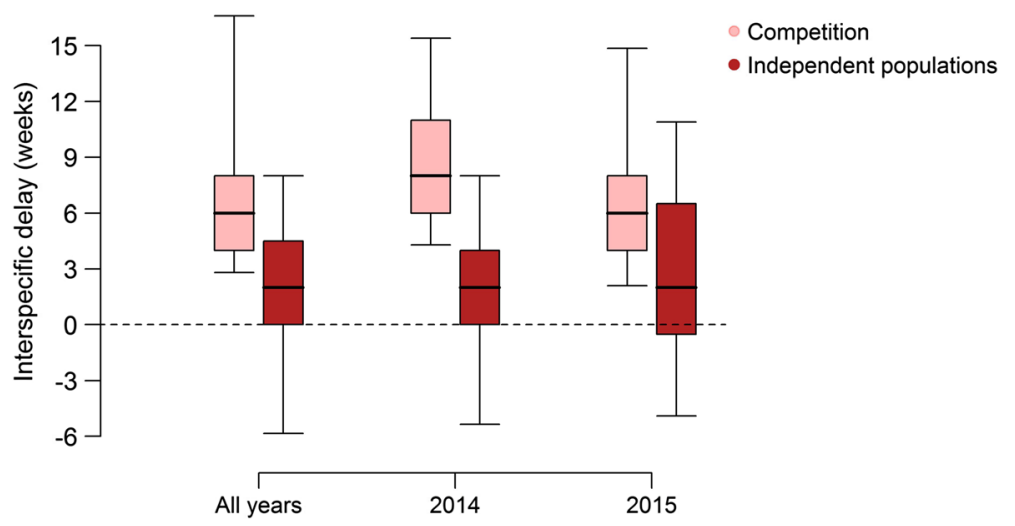

Fig. 6 Interspecific delay by selected model. The interspecific delay (in weeks, median, quartiles and 95\% quantiles) computed for Ae. albopictus and Cx. pipiens capture patterns by the selected model. Distributions are shown for all time series combined and aggregated by year 


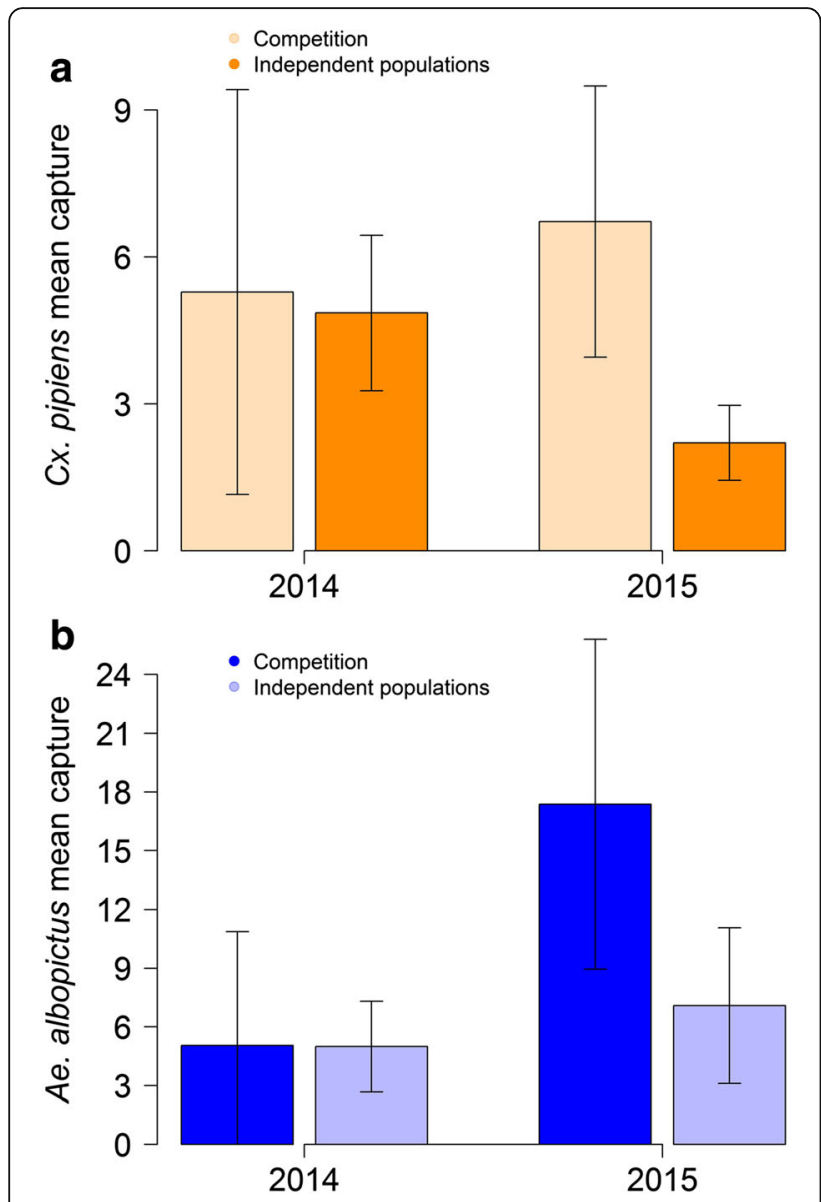

Fig. 7 Average recorded captures by selected model. Average number of captured Cx. pipiens (a) and Ae. albopictus (b) per site by selected model and year. Black lines represent the $95 \%$ confidence intervals

due to competition of about $50 \%$ on average (and up to $70 \%$ in some sites), compared to equivalent environmental conditions where competition was discounted.

Under natural conditions, competition occurs within breeding sites where the two species happen to oviposit together, mainly in urban and peri-urban areas. Adults of Ae. albopictus and Cx. pipiens captured in a given trap emerged from various breeding sites present in the neighbourhood of the trap location. Among these breeding sites, some are colonized by a single larval species, while others are colonized by both species, where they then compete for resources. Therefore, our sampling procedure can only weigh the average effect of competition in the neighbourhood of the trapping site. Our criterion for model selection was designed conservatively to identify sites where competition has a strong overall effect on the temporal patterns of mosquito abundance (see section "Model fit" in the Additional file 1: Database S1). It is likely that competition occurs, to a lower degree, even in trapping sites that were not classified as "competition" sites. To explore the robustness of results with respect to the model selection criterion, we did a sensitivity analysis by using different score functions for model selection and considering different threshold values. Results confirmed our main conclusions and suggested that competition may have shaped Cx. pipiens dynamics in 30 to $50 \%$ of all time series (see section "DIC and AIC analysis" in the Additional file 1: Database 1 for details).

For the sake of simplicity, in this study we assumed competition would affect only $C x$. pipiens larval survival. It has been shown that interspecific competition at this stage might also increase $C x$. pipiens larval developmental time [28] and reduce the body size of newly emerged adults $[21,27]$, with possible negative implications for female fecundity and longevity [21]. Moreover, the body size has been associated with female susceptibility to virus infection $[16,19]$, and female longevity is a main component of the vectorial capacity. Non-lethal effects are more likely to have an impact at low densities of Ae. albopictus larvae $[27,28]$, i.e. in situations where the independent population hypothesis could not be rejected by the present analysis, or at poor food resource conditions [21]. Thus, the inclusion of non-lethal effects in the competition model might unveil an even more widespread importance of competition effects on vector populations and arbovirus transmission. However, abundance data provided by mosquito trapping only give information on the cumulative effects of competition on the adult population; therefore, they are insufficient to distinguish the relative contribution of different competition effects. We chose to only model increased larval mortality, being the strongest competition outcome $[27,28]$ and the one most directly affecting the adult population. Nonetheless, quantitative experiments collecting further data on non-lethal competition effects might improve the development of models and foster our understanding of ecological mechanisms. In addition, given the importance of climatic factors in shaping mosquito population dynamics, important additional insights will be needed, from further experiments, to quantify the effect of temperature on the strength of lethal and nonlethal effects of interspecific competition [27].

\section{Conclusions}

We found that interspecific competition between $C x$. pipiens and Ae. albopictus is common in temperate climates and it is enhanced by higher mosquito densities produced by higher temperatures. Drier weather conditions may also induce a higher overlap of breeding sites for different mosquito species, increasing the overall chances for competition. We have shown that competition amplifies the temporal separation between seasonal patterns of the two species, with $C x$. pipiens arising early and declining more quickly with the rise of Ae. albopictus. Finally, we have shown that competition induces significant reductions in the total abundance of $C x$. pipiens. 
Understanding the interaction between climatic variables, competition and resulting vector abundances can be important to improve our estimates of epidemiological risks for arboviruses for which $C x$. pipiens and Ae. albopictus are competent vectors, and for the assessment of vector control strategies [57, 58]. Furthermore, recent findings have shown that interspecific competition at the larval stage may strongly affect the viral competence of adult mosquitoes [16] as well as their vectorial capacity by modifying their longevity $[20,21]$. If similar effects exist in the competition between $A e$. albopictus and $C x$. pipiens, they would significantly impact the viral susceptibility and transmission potential of local mosquito populations and should therefore be considered in the estimation of outbreak risks $[52,53]$.

\section{Additional files}

\section{Additional file 1: Database S1. Supporting text containing methodological details and additional results. (PDF 872 kb)}

Additional file 2: Database S2. Observed time series. Datasets used for model calibration: trapped adult mosquitoes of both species. (XLSX $18 \mathrm{~kb}$ )

Additional file 3: Figure S1. Model fit for Cx. pipiens (orange) and Ae. albopictus (b/ue) in the 73 datasets. Left side: datasets from 2014; sites where the competition model was selected are shown in the first two rows. Right side: datasets from 2015; sites where the competition model was selected are shown in the first six rows. Squares: capture data; lines and shaded areas: posterior model estimates with 95\% credible intervals. (JPG 19486 kb)

\section{Acknowledgements}

We thank Francesca Bussola, Charlotte Lapère, Sara Carlin, Graziana Da Rold Marco Dal Pont, Nicola Ferro Milone, Luca Tripepi, for mosquito collection and Markus Neteler and Markus Metz for providing Land Surface Temperature data. Authors wish to thank Joanne Lello for her help in the proofreading of the manuscript.

\section{Funding}

This work was funded by the Autonomous Province of Trento (Italy), Research funds for Grandi Progetti (Prot. n. 319292-S116-2013), Project LExEM (Laboratory of excellence for epidemiology and modelling, http://www.lexem.eu). The funders had no role in study design, data collection and analysis, or preparation of the manuscript.

\section{Availability of data and materials}

All data generated or analysed during this study are included in this published article and its additional files.

\section{Authors' contributions}

GM, GG, SM and RR conceived the study, and participated in its design and coordination. GM and GG performed model simulations and the statistical analysis and drafted the manuscript. FB, DA, GC, FM coordinated the mosquito collections and built the original dataset. FB, GC, FM and AR contributed to interpretation and critical review. All authors read and approved the final manuscript.

\section{Competing interests}

The authors declare that they have no competing interests.

\section{Consent for publication}

Not applicable.

Ethics approval and consent to participate Not applicable.

\section{Author details}

${ }^{1}$ Department of Mathematics, University of Trento, Trento, Italy. ${ }^{2}$ Department of Biodiversity and Molecular Ecology, Research and Innovation Centre, Fondazione Edmund Mach, San Michele all'Adige, Trento, Italy. ${ }^{3}$ Fondazione Bruno Kessler, Trento, Italy. ${ }^{4}$ Laboratory of Parasitology, Istituto Zooprofilattico Sperimentale delle Venezie, Padova, Italy.

Received: 22 November 2016 Accepted: 16 February 2017

Published online: 23 February 2017

\section{References}

1. Tilman D. Resource competition and community structure. Princeton: Princeton University Press; 1982.

2. Leisnham PT, LaDeau SL, Juliano SA. Spatial and temporal habitat segregation of mosquitoes in urban Florida. PLoS One. 2014;9(3):e91655.

3. Juliano SA. Species interactions among larval mosquitoes: context dependence across habitat gradients. Annu Rev Entomol. 2009;54:37-56.

4. Farajollahi A, Fonseca DM, Kramer LD, Kilpatrick AM. "Bird biting" mosquitoes and human disease: a review of the role of Culex pipiens complex mosquitoes in epidemiology. Infect Genet Evol. 2011;11(7):1577-85.

5. Schaffner F, Medlock JM, Bortel W. Public health significance of invasive mosquitoes in Europe. Clin Microbiol Infect. 2013;19(8):685-92.

6. Medlock JM, Hansford KM, Schaffner F, Versteirt V, Hendrickx G, Zeller H, et al. A review of the invasive mosquitoes in Europe: ecology, public health risks, and control options. Vector Borne Zoonotic Dis. 2012;12(6):435-47.

7. Chouin-Carneiro T, Vega-Rua A, Vazeille M, Yebakima A, Girod R, Goindin D, et al. Differential susceptibilities of Aedes aegypti and Aedes albopictus from the Americas to Zika virus. PLoS Negl Trop Dis. 2016;10(3):e0004543.

8. Fortuna C, Remoli ME, Severini F, Di Luca M, Toma L, Fois F, et al. Evaluation of vector competence for West Nile virus in Italian Stegomyia albopicta (=Aedes albopictus) mosquitoes. Med Vet Entomol. 2015;29(4):430-3.

9. Paupy C, Delatte H, Bagny L, Corbel V, Fontenille D. Aedes albopictus, an arbovirus vector: from the darkness to the light. Microb Infect. 2009;11 (14-15):1177-85.

10. Vega-Rua A, Zouache K, Caro V, Diancourt L, Delaunay P, Grandadam M, et al. High efficiency of temperate Aedes albopictus to transmit chikungunya and dengue viruses in the southeast of France. PLoS One. 2013;8(3):e59716.

11. Vega-Rúa A, Zouache K, Girod R, Failloux A-B, Lourenço-de-Oliveira R. High level of vector competence of Aedes aegypti and Aedes albopictus from ten American countries as a crucial factor in the spread of chikungunya virus. J Virol. 2014:88(11):6294-306.

12. Wong P-SJ, Li MI, Chong C-S, Ng L-C, Tan C-H. Aedes (Stegomyia) albopictus (Skuse): a potential vector of Zika virus in Singapore. PLoS Negl Trop Dis. 2013;7(8):e2348

13. Zeller HG, Schuffenecker I. West Nile virus: an overview of its spread in Europe and the Mediterranean Basin in contrast to its spread in the Americas. Eur J Clin Microbiol Infect Dis. 2004;23(3):147-56.

14. Colborn JM, Smith KA, Townsend J, Damian D, Nasci RS, Mutebi J-P. West Nile virus outbreak in Phoenix, Arizona-2010: entomological observations and epidemiological correlations. J Am Mosq Control Assoc. 2013;29(2):123-32.

15. Kilpatrick AM, Pape WJ. Predicting human West Nile virus infections with mosquito surveillance data. Am J Epidemiol. 2013;178(5):829-35.

16. Alto BW, Lounibos LP. Vector competence for arboviruses in relation to the larval environment of mosquitoes. In: Takken W, Koenraadt CJM, editors. Ecology of parasite-vector interactions. Wageningen: Wageningen Academic Publishers; 2013. p. 81-101.

17. Alto BW, Lounibos LP, Higgs S, Juliano SA. Larval competition differentially affects arbovirus infection in Aedes mosquitoes. Ecology. 2005;86(12):3279-88.

18. Alto BW, Lounibos LP, Mores CN, Reiskind MH. Larval competition alters susceptibility of adult Aedes mosquitoes to dengue infection. Proc R Soc B. 2008;275(1633):463-71.

19. Bevins SN. Invasive mosquitoes, larval competition, and indirect effects on the vector competence of native mosquito species (Diptera: Culicidae). Biol Invasions. 2007;10(7):1109-17.

20. Reiskind MH, Lounibos LP. Effects of intraspecific larval competition on adult longevity in the mosquitoes Aedes aegypti and Aedes albopictus. Med Vet Entomol. 2009;23(1):62-8.

21. Costanzo KS, Muturi EJ, Lampman RL, Alto BW. The effects of resource type and ratio on competition with Aedes albopictus and Culex pipiens (Diptera: Culicidae). J Med Entomol. 2011;48(1):29-38. 
22. Murrell EG, Juliano SA. Detritus type alters the outcome of interspecific competition between Aedes aegypti and Aedes albopictus (Diptera: Culicidae). J Med Entomol. 2008;45:375-83.

23. O'Neal PA, Juliano SA. Seasonal variation in competition and coexistence of Aedes mosquitoes: stabilizing effects of egg mortality or equalizing effects of resources? J Anim Ecol. 2013;82:256-65.

24. Armistead JS, Arias JR, Nishimura N, Lounibos LP. Interspecific larval competition between Aedes albopictus and Aedes japonicus (Diptera: Culicidae) in northern Virginia. J Med Entomol. 2008;45:629-37.

25. Livdahl TP, Willey MS. Prospects for an invasion: competition between Aedes albopictus and native Aedes triseriatus. Science. 1991;253:189-91.

26. Novak MG, Higley LG, Christianssen CA, Rowley WA. Evaluating larval competition between Aedes albopictus and A. triseriatus (Diptera: Culicidae) through replacement series experiments. Environ Entomol. 1993;22:311-8.

27. Carrieri M, Bacchi M, Bellini R, Maini S. On the competition occurring between Aedes albopictus and Culex pipiens (Diptera: Culicidae) in Italy. Environ Entomol. 2003:32:1313-21.

28. Costanzo KS, Mormann K, Juliano SA. Asymmetrical competition and patterns of abundance of Aedes albopictus and Culex pipiens (Diptera: Culicidae). J Med Entomol. 2005;42:559-70.

29. Becker N, Petric D, Zgomba M, Boase C, Madon M, Dahl C, et al. Mosquitoes and their control. Berlin: Springer, Berlin Heidelberg; 2010.

30. Roiz D, Neteler M, Castellani C, Arnoldi D, Rizzoli A. Climatic factors driving invasion of the tiger mosquito (Aedes albopictus) into new areas of Trentino, northern Italy. PLoS One. 2011;6:e14800.

31. Rosa R, Marini G, Bolzoni L, Neteler M, Metz M, Delucchi L, et al. Early warning of West Nile virus mosquito vector: climate and land use models successfully explain phenology and abundance of Culex pipiens mosquitoes in north-western Italy. Parasit Vectors. 2014;7:269.

32. Verna TN. Species composition and seasonal distribution of mosquito larvae (Diptera: Culicidae) in southern New Jersey, Burlington County. J Med Entomol. 2015;52:1165-9.

33. Denlinger DL, Armbruster PA. Mosquito diapause. Annu Rev Entomol. 2014; 59:73-93.

34. Spielman A, Wong J. Environmental control of ovarian diapause in Culex pipiens. Ann Entomol Soc Am. 1973;66:905-7.

35. Ciota AT, Matacchiero AC, Kilpatrick AM, Kramer LD. The effect of temperature on life history traits of Culex mosquitoes. J Med Entomol. 2014;51:55-62.

36. Ruybal JE, Kramer LD, Kilpatrick AM. Geographic variation in the response of Culex pipiens life history traits to temperature. Parasit Vectors. 2016;9:116.

37. Delatte H, Gimonneau G, Triboire A, Fontenille D. Influence of temperature on immature development, survival, longevity, fecundity, and gonotrophic cycles of Aedes albopictus, vector of chikungunya and dengue in the Indian Ocean. J Med Entomol. 2009:46:33-41.

38. Erickson RA, Presley SM, Allen LJS, Long KR, Cox SB. A stage-structured, Aedes albopictus population model. Ecol Modell. 2010;221:1273-82.

39. Poletti P, Messeri G, Ajelli M, Vallorani R, Rizzo C, Merler S. Transmission potential of chikungunya virus and control measures: the case of Italy. PLoS One. 2011;6:e18860.

40. Tran A, L'Ambert G, Lacour G, Benoît R, Demarchi M, Cros M, et al. A rainfalland temperature-driven abundance model for Aedes albopictus populations. Int J Environ Res Public Health. 2013;10:1698-719.

41. Gong H, DeGaetano AT, Harrington LC. Climate-based models for West Nile Culex mosquito vectors in the Northeastern US. Int J Biometeorol. 2011;55: 435-46

42. Lončarić Ž, K Hackenberger B. Stage and age structured Aedes vexans and Culex pipiens (Diptera: Culicidae) climate-dependent matrix population model. Theor Popul Biol. 2013;83:82-94.

43. Marini G, Poletti P, Giacobini M, Pugliese A, Merler S, Rosà R. The role of climatic and density dependent factors in shaping mosquito population dynamics: the case of Culex pipiens in northwestern Italy. PLoS One. 2016;11: e0154018

44. Neteler M, Roiz D, Rocchini D, Castellani C, Rizzoli A. Terra and Aqua satellites track tiger mosquito invasion: modelling the potential distribution of Aedes albopictus in north-eastern Italy. Int J Health Geogr. 2011;10:49.

45. Lühken R, Pfitzner WP, Börstler J, Garms R, Huber K, Schork N, et al. Field evaluation of four widely used mosquito traps in Central Europe. Parasit Vectors. 2014;7:268.

46. Severini F, Toma L, Di Luca M, Romi R. Le zanzare italiane: generalità e identificazione degli adulti (Diptera, Culicidae). Fragm Entomol. 2009;41:213.
47. Jacovitti G, Scarano G. Discrete time techniques for time delay estimation. IEEE Trans Signal Process. 1993;41:525-33.

48. Wan Z. New refinements and validation of the collection-6 MODIS land-surface temperature/emissivity product. Remote Sens Environ. 2014;140:36-45.

49. Metz M, Rocchini D, Neteler M. Surface temperatures at the continental scale: tracking changes with remote sensing at unprecedented detail. Remote Sens. 2014;6:3822-40.

50. Meteotrentino. http://www.meteotrentino.it. Accessed 16 Nov 2016

51. Naval Oceanography Portal: Data Services (2016) United States Naval Meteorology and Oceanography Command. http://www.usno.navy.mil/USNO/ astronomical-applications/data-services/data-services. Accessed 15 June 2016.

52. Guzzetta G, Montarsi F, Baldacchino FA, Metz M, Capelli G, Rizzoli A, et al. Potential risk of dengue and chikungunya outbreaks in northern Italy based on a population model of Aedes albopictus (Diptera: Culicidae). PLoS Negl Trop Dis. 2016;10:e0004762.

53. Guzzetta G, Poletti P, Montarsi F, Baldacchino F, Capelli G, Rizzoli A, et al. Assessing the potential risk of Zika virus epidemics in temperate areas with established Aedes albopictus populations. Euro Surveill. 2016;21(15).

54. Gilks WR, Richardson S, Spiegelhalter D. Markov Chain Monte Carlo in practice. London: Chapman \& Hall; 1996.

55. Spiegelhalter DJ, Best NG, Carlin BR, van der Linde A. Bayesian measures of model complexity and fit. J R Stat Soc Series B Stat Methodol. 2002;64:583-616.

56. Spiegelhalter DJ, Best NG, Carlin BP, van der Linde A. The deviance information criterion: 12 years on. J R Stat Soc Series B Stat Methodol. 2014; 76:485-93.

57. Baldacchino F, Caputo B, Chandre F, Drago A, della Torre A, Montarsi F, et al. Control methods against invasive Aedes mosquitoes in Europe: a review. Pest Manag Sci. 2015;71:1471-85.

58. Bellini R, Zeller $H$, Van Bortel W. A review of the vector management methods to prevent and control outbreaks of West Nile virus infection and the challenge for Europe. Parasit Vectors. 2014;7:323.

\section{Submit your next manuscript to BioMed Central and we will help you at every step:}

- We accept pre-submission inquiries

- Our selector tool helps you to find the most relevant journal

- We provide round the clock customer support

- Convenient online submission

- Thorough peer review

- Inclusion in PubMed and all major indexing services

- Maximum visibility for your research

Submit your manuscript at www.biomedcentral.com/submit
) Biomed Central 